\title{
Application of a Semi-in-Lens FE-SEM to the Crystallographic Analysis with the EBSD Technique
}

Hiroyuki Ito ${ }^{1}$, Yoichiro Hashimoto ${ }^{1}$, Shuichi Takeuchi ${ }^{1}$, Masahiro Sasajima ${ }^{2}$, Hirofumi Sato ${ }^{2}$, Hirobumi Morita ${ }^{3}$

1. Application Development Department, Science Systems Design Division, Hitachi High-Technologies, 1040, Ichige, Hitachinaka, Ibaraki, 312-0033, Japan

2. Electron Microscope Systems Design 1st Department, Science Systems Design Division, Hitachi

High-Technologies, 882, Ichige, Hitachinaka, Ibaraki, 312-8504, Japan

3. NanoAnalysis Department, Oxford Instruments, IS Building, 3-32-42, Higashi-Shinagawa, Shinagawa-ku, Tokyo, 140-0002, Japan

An FE-SEM is a powerful tool by its high resolution and high performance and is utilized widely in various fields of study. The electron back-scattered diffraction (EBSD) using a FE-SEM is one of the useful methods for the determination of crystallographic characteristics such as direction, symmetry, etc. of the specimen. In general, an out-lens type SEM is good for EBSD because the objective lens field is kept within the lens structure and the magnetic field will not conflict with the EBSD signal. Recently, in the field of material sciences the demand of the EBSD analysis of fine crystals increases.

To satisfy both the image quality and the EBSD performance, we examined the EBSD analysis using a semi-in-lens cold FEG-SEM. Figure 1 is a general view of the SU8240 (a) and a high resolution image of meso-porous silica (b). The SU8200 series has various signal detection system, in which various kinds of signals such as SE, high and low angle BSE are simultaneously taken at the wide ranges of acceleration voltage and utilized them for characterization of samples [1].

In this study, Si (001) sample was used for a fundamental experiment. The EBSD was obtained with an Aztec-HKL EBSD system equipped with the distortion correcting function of EBSD pattern (Magnetic Field Correction: MFC). We examined the stability of the cold FEG for EBSD analysis and the degree of distortion of EBSD patterns compared with the data from out-lens FE-SEM.

Figure 2 shows the relationship between working distance and the distortion of the EBSD patterns. The value of the distortion of EBSD patterns without correction became small as the working distance become longer, resulting in 5 degrees at $25 \mathrm{~mm}$ working distance. With correction, the distortion was within 2 degrees at all working distances. This indicates that MFC is useful to obtain the precise EBSD pattern and high resolution images. Also, this is consistent with the notion of the semi-in-lens field no longer enveloping the sample at the longer working distances. And then, the MFC correction is available to obtain the precise EBSD patterns. In order to examine the stability of cold FEG for EBSD analysis, we acquired EBSD map of Si (001) for 3 hours. Then, we extracted monochrome graduation of each time from band contrast map, and plotted it. The band contrast is expressed the intensity of the EBSD pattern in monochrome graduation and has value from 0 to 255 . If the probe current greatly drops, the band contrast will drop as well. As shown in Figure3, the change of image contrast with the time process was almost flat and stable. The EBSD pattern intensity was stable to within $6 \%$ and the software was able to accurately index the pattern throughout the acquisition period.

Figure 4 is an application of EBSD analysis of gold crystal by SU8240. Herein, fine grains were clearly 
captured with contour lines (a). As shown in IPF maps at $\mathrm{z}$ (b) and $\mathrm{x}$ (c) directions, the crystal directions grains smaller than $50 \mathrm{~nm}$ were clearly visualized. These results suggest that a semi-in-lens SEM is available to the EBSD analysis of fine grains with MFC system.

References:

[1] S Takeuchi et al, Microsc. Microanal. Vol.19(Suppl 2) (2013) p.p.1310-1311.

(a)

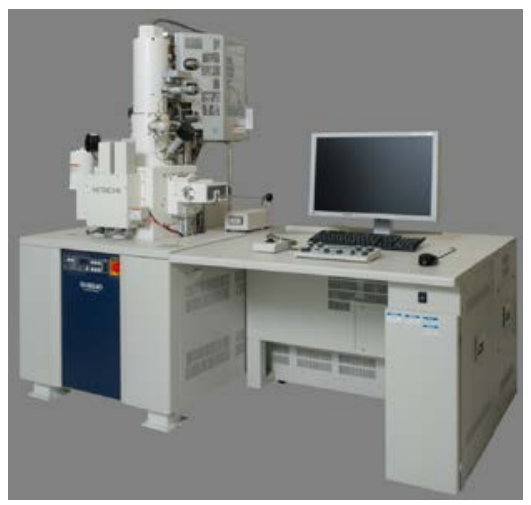

(b)

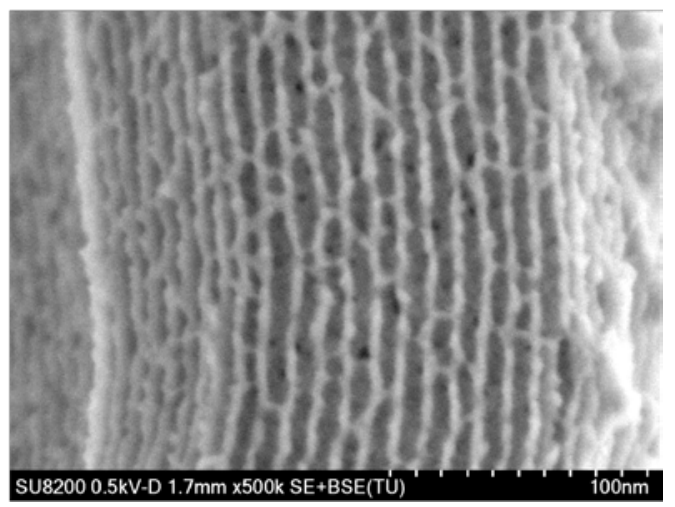

Figure 1. General view of the SU8240 and high resolution image taken by the SU8200

(a)General view of the SU8240, (b) High resolution image of mesoporous silica

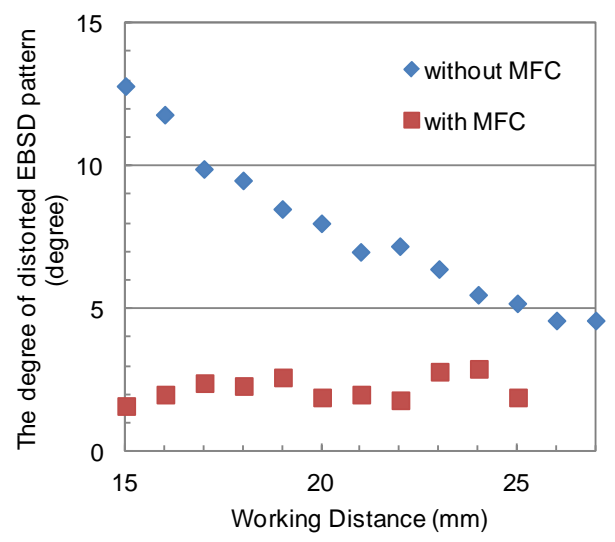

Figure 2. The relationship between working distance and distorted EBSD pattern angle

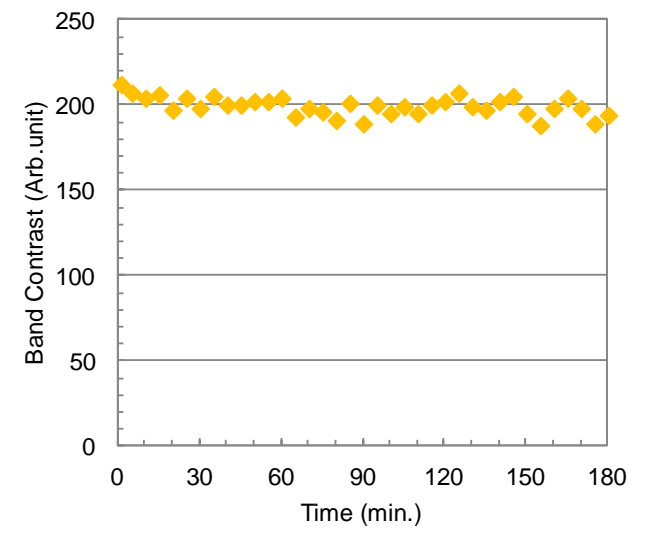

Figure 3. The change of band contrast (a)

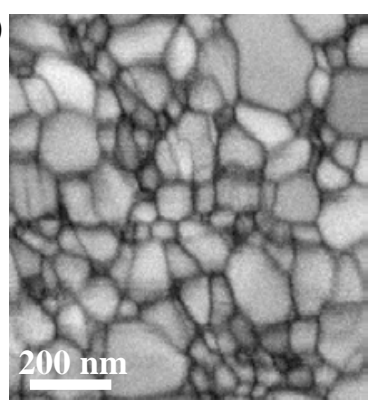

(b)

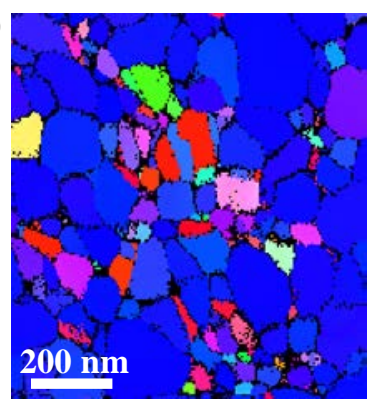

(c)

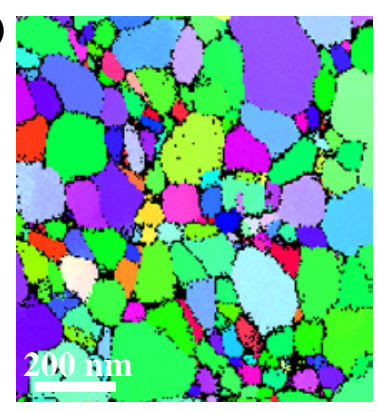

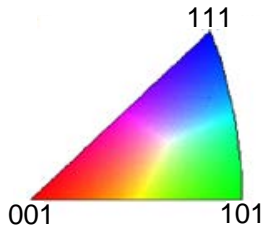

Figure 4. EBSD map of fine gold grain sample

(a) Band contrast map, (b)IPF map(Z), (c)IPF map(X) 